\title{
Label Cover Instances with Large Girth and the Hardness of Approximating Basic $k$-Spanner
}

\author{
MICHAEL DINITZ, Weizmann Institute of Science \\ GUY KORTSARZ, Rutgers University-Camden \\ RAN RAZ, Weizmann Institute of Science
}

\begin{abstract}
We study the well-known Label Cover problem under the additional requirement that problem instances have large girth. We show that if the girth is some $k$, the problem is roughly $2^{\left(\log ^{1-\epsilon} n\right) / k}$ hard to approximate for all constant $\epsilon>0$. A similar theorem was claimed by Elkin and Peleg [2000] as part of an attempt to prove hardness for the basic $k$-spanner problem, but their proof was later found to have a fundamental error. Thus, we give both the first nontrivial lower bound for the problem of Label Cover with large girth as well as the first full proof of strong hardness for the basic $k$-spanner problem, which is both the simplest problem in graph spanners and one of the few for which super-logarithmic hardness was not known. Assuming $N P \nsubseteq B P T I M E\left(2^{p o l y l o g}(n)\right)$, we show (roughly) that for every $k \geq 3$ and every constant $\epsilon>0$, it is hard to approximate the basic $k$-spanner problem within a factor better than $2^{\left(\log ^{1-\epsilon} n\right) / k}$. This improves over the previous best lower bound of only $\Omega(\log n) / k$ from Kortsarz [2001]. Our main technique is subsampling the edges of 2-query probabilistically checkable proofs (PCPs), which allows us to reduce the degree of a PCP to be essentially equal to the soundness desired. This turns out to be enough to basically guarantee large girth.

CCS Concepts: • Theory of computation $\rightarrow$ Interactive proof systems; Routing and network design problems
\end{abstract}

Additional Key Words and Phrases: Graph spanners, probabilistically checkable proofs

\section{ACM Reference Format:}

Michael Dinitz, Guy Kortsarz, and Ran Raz. 2015. Label Cover instances with large girth and the hardness of approximating basic $k$-spanner. ACM Trans. Algorithms 12, 2, Article 25 (December 2015), 16 pages.

DOI: http://dx.doi.org/10.1145/2818375

\section{INTRODUCTION}

In this article, we deal with 2-query probabilistically checkable proofs (PCPs) and variants of the Label Cover problem. Label Cover was originally defined by Arora and Lund in their early survey on hardness of approximation [Arora and Lund 1997]. Since then, Label Cover has been widely used as a starting point when proving hardness of approximation, as it corresponds naturally to 2-query PCPs and one-round twoprover interactive proof systems. Notable examples are the reduction to the Set Cover problem [Lund and Yannakakis 1994; Feige 1998], the reduction to the maximum

M. Dinitz was supported by the Israel Science Foundation under grant 452/08, the U.S.-Israel Binational Science Foundation under grant 2010418, and a Minerva grant. G. Kortsarz was supported by the National Science Foundation under grant 434923. R. Raz was supported by an Israel Science Foundation grant and by the I-CORE Program of the Planning and Budgeting Committee.

Authors' addresses: M. Dinitz, Department of Computer Science, Johns Hopkins University, 160 Malone Hall, 3400 N Charles St, Baltimore, MD 21218; email: mdinitz@cs.jhu.edu; G. Kortsarz, Department of Computer Science, BSB Room 322, 227 Penn Street, Rutgers University, Camden, NJ 08102; email: guyk@ camden.rutgers.edu; R. Raz, Faculty of Mathematics and Computer Science, The Weizmann Institute of Science, Rehovot 76100, Israel; email: ran.raz@weizmann.ac.il.

Permission to make digital or hard copies of all or part of this work for personal or classroom use is granted without fee provided that copies are not made or distributed for profit or commercial advantage and that copies bear this notice and the full citation on the first page. Copyrights for components of this work owned by others than the author(s) must be honored. Abstracting with credit is permitted. To copy otherwise, or republish, to post on servers or to redistribute to lists, requires prior specific permission and/or a fee. Request permissions from permissions@acm.org.

2015 Copyright is held by the owner/author(s). Publication rights licensed to ACM.

ACM 1549-6325/2015/12-ART25 \$15.00

DOI: http://dx.doi.org/10.1145/2818375 
independent set problem [Lund and Yannakakis 1994; Håstad 2001], and the reduction to the minimum coloring problem [Feige and Kilian 1998]. Certain reductions from Label Cover, however, require special properties of the Label Cover instances. So then the question becomes: is Label Cover still hard even when restricted to these instances? For example, the famous Unique Games Conjecture of Khot [2005] can be thought of as a conjecture that Label Cover is still difficult to approximate when the relation on each edge is required to be a bijection.

A different type of requirement is on the structure of the Label Cover graph rather than on the allowed relations. Elkin and Peleg [2000] introduced a restriction of Label Cover (actually, a slight variant of Label Cover known as Min-Rep) to graphs with large girth and proved that if Label Cover is hard even on these graphs, then the basic $k$ spanner problem is also hard to approximate. They then gave a proof that Label Cover is indeed hard to approximate on large-girth graphs, but unfortunately this proof was later found to have a flaw (as Elkin and Peleg point out in Section 1.3 of Elkin and Peleg [2007]). In this article, we give a completely new proof that Label Cover and Min-Rep are hard to approximate on large-girth graphs. Our argument is based on subsampling edges of a 2-query PCP/Label Cover instance. Subsampling of 2-query PCPs and Label Cover instances has been done before for other reasons (e.g., see Goldreich and Sudan [2006]), but we show that the sampling probability can be set low enough to destroy most small cycles while still preserving hardness, and this technique was not previously used in this context. Remaining cycles can then be removed deterministically.

\subsection{Label Cover and Probabilistically Checkable Proofs}

A PCP is a string (proof) together with a verifier algorithm that checks the proof (probabilistically). There are several important parameters of a PCP, including the following:

(1) Completeness (c): The minimum probability that the verifier accepts a correct proof. All PCPs in this article have completeness 1.

(2) Soundness (or error) ( $s$ ): The maximum probability that the verifier accepts a proof of an incorrect claim.

(3) Queries: The number of queries that the verifier makes to the proof. In this article, we will study the case when the verifier only makes two queries.

(4) Size: The number of positions in the proof (i.e., the length).

(5) Alphabet $(\Sigma)$ : The set of symbols in which the proof is written. We will only be concerned with PCPs for which $|\Sigma|$ is at most polynomial in the size of the PCP, so we will assume this throughout.

For this article, we will be concerned with 2-query PCPs, which are the special case when the verifier is only allowed to query two positions of the proof. We will also assume (without loss of generality) that these two queries are to different parts of the proof-for instance, there is some set of positions $A$ that can be read by the first query and some other set of positions $B$ that can be read by the second query with $A \cap B=\emptyset$.

For this type of PCP, there are two natural graphs that represent it. The first (and simpler), which is sometimes called the supergraph, is a bipartite graph $(A, B, E)$ in which there is a vertex for every position of the proof and an edge between two positions if there is a possibility that the verifier might query those two positions. By our assumption, this graph is bipartite. We also assume that the verifier chooses its query uniformly at random from these edges, which is in fact the case in the PCPs that we will use (particularly in the PCP for Max-3SAT(5) obtained by parallel repetition). Vertices of this graph will sometimes be called supervertices, and edges will be superedges.

The second graph can be thought of as an expansion of the supergraph in which the test the verifier does is explicitly contained in the graph. This graph is also bipartite, 
with vertex set $\left(A \times \Sigma_{A}, B \times \Sigma_{B}\right)$, where $\Sigma_{A}$ is the alphabet used in $A$ positions of the proof and similarly for $\Sigma_{B}$. There is an edge between vertices $(a, \alpha)$ and $(b, \beta)$ if the verifier might query $a$ and $b$ together (i.e., $(a, b)$ is an edge in the supergraph) and if, upon such queries, the verifier will accept the proof if it sees values $\alpha$ in position $a$ and $\beta$ in position $b$. We call this graph the Min-Rep graph. This is related to the work in Kortsarz [2001].

There is a natural correspondence between these types of PCPs and the optimization problem of Label Cover. In Label Cover, we are given a bipartite graph $G=(A, B, E)$, alphabets $\Sigma_{A}$ and $\Sigma_{B}$, and for every edge $e \in E$ a nonempty relation $\pi_{e} \subseteq \Sigma_{A} \times \Sigma_{B}$. The goal is to find assignments $\gamma_{A}: A \rightarrow \Sigma_{A}$ and $\gamma_{B}: B \rightarrow \Sigma_{B}$ to maximize the number of edges $e=(a, b)$ for which $\left(\gamma_{A}(a), \gamma_{B}(b)\right) \in \pi_{e}$ (in which case we say that the edge is satisfied or covered). It is easy to see that the existence of PCPs for NP-hard problems implies that Label Cover is hard to approximate: in particular, if we use the supergraph and set the relation to be answers on which the verifier accepts, then it is hard to distinguish instances in which at least a $c$ fraction of the edges are satisfiable (a valid proof) from instances in which at most an $s$ fraction of the edges are satisfiable (an invalid proof). The exact nature of the hardness assumption is based on the size of the PCP: if it has size $m(n)$ (where $n$ is the size of the original problem instance), then the hardness assumption is that NP is not contained in DTIME $(\operatorname{pol} y(m(n)))$ (for deterministic PCP constructions and approximation algorithms) or BPTIME $(\operatorname{pol} y(m(n)))$ (for randomized PCP constructions or approximation algorithms). We let Label Cover $_{k}$ be the Label Cover problem with the additional restriction that the girth of $G$ is larger than $k$.

We now describe a slight variant of Label Cover known as Min-Rep (originally defined by Kortsarz [2001]) that has been useful for proving hardness of approximation for network design problems such as spanners. It can be thought of as a minimization version of Label Cover with the additional property that the alphabets are represented explicitly as vertices in a graph. Consider the Min-Rep graph $G^{\prime}=\left(A \times \Sigma_{A}, B \times \Sigma_{B}, E^{\prime}\right)$. For every $i \in A$, let $A_{i}=\left\{(i, \alpha) \in A \times \Sigma_{A}\right\}$ be the set of vertices in the Min-Rep graph corresponding to vertex $i$ in the Label Cover graph, and similarly for $i \in B$, let $B_{i}=\left\{(i, \beta) \in B \times \Sigma_{B}\right\}$. Our goal is to choose a set $S$ of vertices of $G^{\prime}$ of minimum size so that for every $(i, j) \in E$, there are vertices $(i, \alpha)$ and $(j, \beta)$ in $S$ that are joined by an edge in $E^{\prime}$. Such a set is called a $R E P$-cover, and the vertices in it are called representatives. Less formally, we can think of Min-Rep as being the problem of assigning to every vertex in the supergraph a set of labels/representatives (unlike Label Cover, in which only a single label is assigned) so that for every superedge $(a, b)$, there is at least one label assigned to $a$ and at least one label assigned to $b$ that satisfy the relation $\pi_{(a, b)}$. Note that in the Min-Rep graph, the number of vertices is $|A| \cdot\left|\Sigma_{A}\right|+|B| \cdot\left|\Sigma_{B}\right|$, which means that the size of a Min-Rep instance might be larger than the size of the associated Label Cover instance. The supergirth of a Min-Rep graph is just the girth of the supergraph (i.e., the girth of the associated Label Cover instance). As with Label Cover, we let Min-Rep $e_{k}$ be the Min-Rep problem with the additional restriction that the supergirth is larger than $k$.

Two parameters of PCPs/Label Cover that will be important for us are the degree and the girth. The degree of a PCP is the maximum degree of a vertex in the supergraph/associated Label Cover instance. Similarly, the girth of a PCP is the girth in the supergraph/associated Label Cover instance (recall that the girth of a graph is the length of the smallest cycle).

\subsection{The Basic $k$-Spanner Problem and Previous Work}

The basic $k$-spanner problem, also called the minimum size $k$-spanner problem, is the second main subject in this article. In this problem, we are given an undirected, unweighted graph $G$ and are asked to find the subgraph $G^{\prime}=\left(V, E^{\prime}\right)$ with the minimum 
number of edges with the property that

$$
\frac{\operatorname{dist}_{G^{\prime}}(u, v)}{\operatorname{dist}_{G}(u, v)} \leq k \text {, for every two vertices } u, v \in V .
$$

In the preceding, the distance between two vertices is just the number of edges in the shortest path between the two vertices, and $d^{2} s_{H}$ is the distance function on a graph $H$. Any subgraph $G^{\prime}$ satisfying (1) is called a $k$-spanner of $G$, and our goal is to find the $k$-spanner with the fewest edges. Elkin and Peleg [2000] proved that if Min-Rep p $_{k+1}$ is hard to approximate, then the basic $k$-spanner problem is also hard to approximate. For completeness, we shall later describe their reduction and proof.

The concept of graph spanners was first invented by Levcopoulos and Lingas [1992] in a geometric context. To the best of our knowledge, the spanner problem on general graphs was first invented indirectly by Peleg and Upfal [1989] in their work on small routing tables. This problem was first explicitly defined in Peleg and Ullman [1989] and Peleg and Schaffer [1989].

Spanners appear in remarkably many applications; the following examples are certainly not exhaustive. Peleg and Upfal [1989] showed an application of spanners for maintaining small routing tables. For further applications toward this subject, see Cowen [2001], Cowen and Wagner [2004], Roddity et al. [2008], and Thorup and Zwick [2001]. In Peleg and Ullman [1989], a relation between sparse spanners and synchronizers for distributed networks was found. In Cohen [1999, 2000], Elkin [2005], and Feigenbaum et al. [2008], applications of spanners to parallel, distributed, and streaming algorithms for shortest paths are described. For applications of spanners to distance oracles, see Baswana and Sen [2006] and Thorup and Zwick [2005]. For applications of spanners in property testing and related subjects, see Bhattacharyya et al. [2009]. See Awerbuch et al. [1992] for an application of spanners in broadcast and Bandelt and Dress [1986] for an application of spanners in biology.

There is also quite a bit known about approximating spanners. For the basic $k$ spanner problem, a paper of Althöfer et al. [1993] shows that every undirected graph on $n$ vertices has a $(2 k-1)$-spanner with at most $n^{1+1 / k}$ edges (for any integer $k \geq 1$ ), which immediately implies an $n^{1 /\lfloor(k+1) / 2\rfloor}$ approximation to basic $k$-spanner when $k \geq 3$. This is because any spanner is a connected graph and hence has at least $n-1$ edges. For $k=2$, no nontrivial absolute bounds are possible, but in terms of approximation, there is an $O(\log n)$ approximation [Kortsarz and Peleg 1994], which is known to be the best possible [Kortsarz 2001]. There are also many spanner variants that have been studied, such as the directed $k$-spanner problem [Dinitz and Krauthgamer 2011a; Berman et al. 2013] and the client-server $k$-spanner problem [Elkin and Peleg 2005]. Essentially all variants are known to be hard to approximate to better than $2^{\log ^{1-\epsilon} n}$ (see Kortsarz [2001] and Elkin and Peleg [2007]), leaving the hardness of the basic version a tantalizing question.

\subsection{Our Results}

All of our results hold for large $n$, so throughout this article we will assume that $n$ is sufficiently large. Our first result is on the hardness of approximating Label Cover with large girth.

THEOREM 1.1. Assuming NP $\nsubseteq B P T I M E\left(2^{\text {polylog(n) }}\right)$, for any constant $\epsilon>0$ and for $3 \leq k \leq \log ^{1-2 \epsilon} n$ there is no polynomial-time algorithm that approximates Label Cover $k$ to a factor better than $2^{\left(\log ^{1-\epsilon} n\right) / k}$. 
We also show how to adapt this hardness from Label Cover to Min-Rep, which then gives us the strong hardness for basic $k$-spanner that originally was claimed by Elkin and Peleg [2000].

THEOREM 1.2. Assuming NP $\nsubseteq B P T I M E\left(2^{\text {polylog(n) }}\right)$, for any constant $\epsilon>0$ and for $3 \leq k \leq \log ^{1-2 \epsilon} n$ there is no polynomial-time algorithm that approximates Min-Rep $p_{k}$ to a factor better than $2^{\left(\log ^{1-\epsilon} n\right) / k}$.

A notable property of our result is that it allows is to improve 2-query PCPs, specifically by decreasing the degree and number of edges of the associated supergraph. This includes, for example, PCPs constructed through the parallel repetition theorem [Raz 1998]. Previously, Goldreich and Sudan [2006] used similar techniques to construct almost-linear size PCPs, but their construction was not applied to almost arbitrary one-round two-prover protocols/2-query PCPs as ours is. Although the hardness for Label Cover with large girth was not known, it seems that this possibility of decreasing the degree was "in the air" among researchers in PCPs. However, this important issue was never explicitly stated in a way that was useful for all researchers interested in lower bounds but rather was only accessible to those who were experts in PCPs. We give a very general theorem, pointing out explicitly the better (smaller) degree and the trade-off with the soundness. Since we make this issue much more explicit, our work has already been used in Laekhanukit [2014] to improve hardness results for vertex connectivity. Indeed, it seems that when using parallel repetition for designing hardness results in the future, our work provides a chance for stronger hardness than a result based solely on Raz [1998]. We expect our work to yield further improved hardness results in the future.

THEOREM 1.3. Assuming NP $\nsubseteq B P T I M E\left(2^{\text {polylog(n)}}\right)$, for any constant $\epsilon>0$ and for $3 \leq k \leq \log ^{1-2 \epsilon} n$ there is no polynomial-time algorithm that approximates the basic $k$-spanner problem to a factor better than $2^{\left(\log ^{1-\epsilon} n\right) / k}$.

This solves an almost 20-year-old open problem posed in Kortsarz and Peleg [1994]. Moreover, as pointed out by Elkin and Peleg [2000], this lower bound is essentially tight when $k=\Theta\left(\log ^{\mu} n\right)$ for constant $0<\mu<1$. This is because Theorem 1.3 implies that in this setting, there is no polynomial-time algorithm that approximates basic $k$-spanner better than $2^{\log ^{1-\mu-\epsilon} n}$, for arbitrarily small constant $\epsilon>0$. On the other hand, in this regime, the trivial upper bound provided by Althöfer et al. [1993] gives an approximation ratio of $2^{O\left(\log ^{1-\mu} n\right)}$.

\subsection{The Error in Elkin and Peleg [2000] and Our Techniques}

To the best of our knowledge, the question answered in Theorem 1.2 regarding the hardness of Min-Rep with large supergirth was first presented by Elkin and Peleg at ICALP 2000. In Elkin and Peleg [2000], the authors tried to create Min-Rep instances with large supergirth that are also hard to approximate, as follows. They started with a 3-Sat(5) instance and associated supergraph, where the supergraph has clauses and variables as vertices, with an edge between a clause and a variable if the variable is in the clause. They then showed how to change the instance to force this graph to have large girth, without losing much in the gap. They then applied the parallel repetition theorem [Raz 1998] and claimed to boost the hardness while maintaining large supergirth. This reduction is incorrect (as Elkin and Peleg acknowledge in Elkin and Peleg [2007]), as nonsimple cycles in the original graph become simple cycles after parallel repetition is applied. In fact, the supergirth in the construction of Elkin 
and Peleg [2000] is 4, no matter what the initial supergirth before parallel repetition is, and thus Elkin and Peleg [2000] does not imply any hardness whatsoever for the large supergirth Min-Rep problem. For the interested reader, in the conference version of Elkin and Peleg [2000], it is Lemma 13 that is incorrect.

Our main idea is to apply parallel repetition first, boosting the gap, and then randomly sample superedges to sparsify the supergraph. It turns out, perhaps surprisingly, that to a certain extent these random choices do not decrease the gap. This may seem nonintuitive at first, as usually the gap is closely related to superdegree and a smaller superdegree would imply a smaller gap. This may have been one of the obstacles in finding a lower bound for Min-Rep with large supergirth. However, it turns out that it is possible to keep the gap despite the smaller superdegree.

The hardness that we derive this way is actually for Label Cover $_{k}$ and not for Min$\operatorname{Rep}_{k}$. The standard reduction from Label Cover to Min-Rep [Kortsarz 2001] entails duplications of many supervertices. This is needed to ensure regularity in the MinRep graph, which is used to ensure that removing a $\mu$ fraction of the supervertices will imply a removal of at most a $\mu$ fraction of the superedges. In Kortsarz [2001], this duplication is done after the parallel repetition step, as the supergirth was not an important quantity. However, such duplications add many cycles of length 4 in the supergraph. We handle this difficulty by performing duplication before we apply parallel repetition.

Regarding the hardness of basic $k$-spanner, in Elkin and Peleg [2000] a reduction is given from Min-Rep $p_{k+1}$ to the basic $k$-spanner problem for $k \geq 3$. This can be viewed as a conditional hardness result: if Min-Rep $\operatorname{R}_{k+1}$ is hard, then so is basic $k$-spanner. By proving hardness for Min-Rep ${ }_{k+1}$, we complete the proof of the hardness of basic $k$-spanner.

The actual situation before our work is as follows. No hardness whatsoever was known for the Min-Rep $k$ problem; our hardness result comes 12 years after this question was first posed. Regarding lower bounds for the basic $k$-spanner problem, this question was first raised in Kortsarz [2001] in APPROX 1998. The best lower bound known (before our work) was $\Omega(\log n) / k$. The improved hardness that we give comes 14 years after this question was first posed.

\section{SAMPLING LEMMA FOR 2-QUERY PCPS}

We begin with our general 2-query PCP sampling lemma. We remark that similar subsampling techniques have been used before (notably by Goldreich and Sudan [2006] to give almost-linear size PCPs), but we specialize the technique with an eye toward giving a trade-off between the soundness and the girth. Since we will only be concerned with regular PCPs, we will phrase it for the special case when the supergraph has $|A|=|B|=n / 2$ and is regular with degree $d$. We will assume without loss of generality that $\left|\Sigma_{A}\right| \geq\left|\Sigma_{B}\right|$. Given such a PCP verifier (i.e., Label Cover instance) $G=(A, B, E)$, let $G_{\alpha}$ be the verifier/instance that we get by subsampling the edges with probability $p_{\alpha}=\frac{\alpha \log \left|\Sigma_{A}\right|}{d}$ (i.e., every edge $e \in E$ is included in $G_{\alpha}$ independently with probability $\left.p_{\alpha}\right)$.

Lemma 2.1. Let $G=(A, B, E)$ be a 2-query PCP verifier/Label Cover instance with completeness 1 and soundness $s$ in which $|A|=|B|=n / 2$, every vertex has degree $d$, and $\left|\Sigma_{A}\right| \geq\left|\Sigma_{B}\right|$. Let $1 \leq \alpha \leq 1 /$ s. Then $G_{\alpha}$ is a PCP verifier with completeness 1 and soundness at most $4 e / \alpha$ with probability at least $1-2^{-n}$.

Proof. It is obvious that $G_{\alpha}$ has completeness 1 with probability 1 , as any valid labeling/proof of $G$ is also valid for $G_{\alpha}$. 
To bound the soundness, first fix a proof / labeling of a NO instance (an incorrect claim). Then by the definition of soundness, we know that in the original verifier, at most an $s$ fraction of the edges are satisfied. After sampling, the expected number of satisfied edges is at most

$$
p_{\alpha} s|E| \leq \frac{|E| \log \left|\Sigma_{A}\right|}{d}=\frac{n}{2} \log \left|\Sigma_{A}\right|
$$

Since the sampling decisions are independent, we can apply a Chernoff bound (e.g., see Theorem 1.1. in Dubhashi and Panconesi), giving us the probability that more than en $\log \left|\Sigma_{A}\right|$ edges are satisfied is at most $2^{-e n \log \left|\Sigma_{A}\right|}=\left|\Sigma_{A}\right|^{-e n}$. But the total number of possible proofs is at most $\left|\Sigma_{A}\right|^{n / 2}\left|\Sigma_{B}\right|^{n / 2} \leq\left|\Sigma_{A}\right|^{n}$. So by a union bound, the probability that any labeling satisfies more than en $\log \left|\Sigma_{A}\right|$ edges is at most $\left|\Sigma_{A}\right|^{-(e-1) n} \leq 2^{-n-1}$. But the expected total number of edges after sampling is $p_{\alpha}|E|=\frac{n}{2} \alpha \log \left|\Sigma_{A}\right|$, and so another Chernoff bound implies that with high probability (at least $1-2^{-n-1}$ ), the number of edges after sampling is at least $(n / 4) \alpha \log \left|\Sigma_{A}\right|$. Thus, with probability at least $1-2^{-n}$, no proof is accepted with probability more than $\left(e n \log \left|\Sigma_{A}\right|\right) /\left((n / 4) \alpha \log \left|\Sigma_{A}\right|\right)=4 e / \alpha$.

Lemma 2.1 shows that we can sample edges so that the average degree is about $\alpha \log |\Sigma|$ without hurting the soundness too much (in particular, the soundness becomes basically $1 / \alpha$ ). Note that if we set $\alpha=1 / s$, this allows us to reduce the average degree to basically $(1 / s) \log \left|\Sigma_{A}\right|$ (a possibly significantly reduction) without affecting the soundness by more than a constant. We would like to claim that this lets us increase the girth, but at this point we will merely prove that any edge is unlikely to be in short cycles. Later we will deterministically remove edges that take part in short cycles, but since that might destroy approximate regularity (which is necessary for our reduction to Min-Rep), we put it off until later.

Lemma 2.2. Fix an edge $(u, v) \in G$. Conditioned on $(u, v) \in G_{\alpha}$, the probability that $(u, v)$ is in a cycle in $G_{\alpha}$ of length at most $k$ is at most $\frac{2\left(\alpha \log \left|\Sigma_{A}\right|\right)^{k-1}}{d}$.

Proof. Let $4 \leq k^{\prime} \leq k$ (note that no edge is in a cycle of length less than 4 in any bipartite graph, including $G)$. Fix a cycle of length $k^{\prime}$ in $G$ that contains $(u, v)$. After conditioning on $(u, v)$ surviving the sampling, the probability that all of the other edges in the cycle are also in $G_{\alpha}$ is $p_{\alpha}^{k^{\prime}-1}=\left(\frac{\alpha \log \left|\Sigma_{A}\right|}{d}\right)^{k^{\prime}-1}$. On the other hand, we know from the degree bound in $G$ that the number of $k^{\prime}$-cycles containing $(u, v)$ is at most $d^{k^{\prime}-2}$. So a union bound implies that the probability that $(u, v)$ is in a $k^{\prime}$-cycle in $G_{\alpha}$ is at most $\frac{\left(\alpha \log \left|\Sigma_{A}\right|\right)^{k^{\prime}-1}}{d}$. Now we take a union bound over all $4 \leq k^{\prime} \leq k$ to get the total probability that $(u, v)$ is in a cycle of length at most $k$ is at most $\sum_{k^{\prime}=4}^{k} \frac{\left(\alpha \log \left|\Sigma_{A}\right|\right)^{k^{\prime}-1}}{d} \leq \frac{2\left(\alpha \log \left|\Sigma_{A}\right|\right)^{k-1}}{d}$ as claimed (assuming without loss of generality that $\alpha \log \left|\Sigma_{A}\right| \geq 2$ ).

It is easy to see that subsampling preserves approximate regularity, but we will now prove so formally.

LEMMA 2.3. If $\alpha \geq 16 \log n$, then with probability at least $1-2 / n$, the degree of every vertex in $G_{\alpha}$ is between $\frac{1}{2} \alpha \log \left|\Sigma_{A}\right|$ and $2 \alpha \log \left|\Sigma_{A}\right|$.

Proof. Since $G$ is regular with degree $d$ and $p_{\alpha}=\frac{\alpha \log \left|\Sigma_{A}\right|}{d}$, the expected degree of a vertex $v$ in $G_{\alpha}$ is clearly $\alpha \log \left|\Sigma_{A}\right|$. So by a Chernoff bound (e.g., see Theorem 1.1. in Dubhashi and Panconesi [2009]), the probability that the degree is less than $1 / 2$ of this or more than twice this is at most $2 \cdot e^{-\alpha \log \left|\Sigma_{A}\right| / 8} \leq 2 /\left|\Sigma_{A}\right|^{-\alpha / 8}$. Since $\alpha \geq 16 \log n$ and $\left|\Sigma_{A}\right| \geq 2$, this probability is at most $2 / n^{2}$, so taking a union bound over vertices implies the probability that all of them have degree in the desired range is at least $1-2 / n$. 


\section{LABEL COVER AND MIN-REP WITH LARGE (SUPER)GIRTH}

In this section, we show that Label Cover and Min-Rep are both hard to approximate, even when restricted to instances with large (super)girth. We start with a PCP verifier with supergraph $G$ and Min-Rep graph $H$, and then use the previously described random sampling technique to get a new supergraph $G_{\alpha}$ and Min-Rep graph $H_{\alpha}$. We now remove from $G_{\alpha}$ any edge that is in a cycle of length at most $k$, giving us a new supergraph $G_{\alpha}^{\prime}$ and Min-Rep graph $H_{\alpha}^{\prime}$ (where an edge $((a, \delta),(b, \beta))$ from $H$ is in $H_{\alpha}^{\prime}$ if $(a, b)$ remains as an edge in $\left.G_{\alpha}^{\prime}\right)$. These instances will form our reduction.

We say that an edge $(i, j) \in G_{\alpha}$ is bad if it is not in $G_{\alpha}^{\prime}$ - that is, it is part of a cycle of length of at most $k$ in $G_{\alpha}$.

Lemma 3.1. Let $16 \log n \leq \alpha \leq \min \left\{1 / s, d^{1 / k} / \log \left|\Sigma_{A}\right|\right\}$. Then with probability larger than $2 / 3$, the number of bad edges is at most $O\left(\frac{n\left(\alpha \log \left|\Sigma_{A}\right|\right)^{k}}{d}\right) \leq O(n)$.

Proof. Lemma 2.2 and Markov's inequality imply that with probability at least 3/4, at most a $\frac{8\left(\alpha \log \left|\Sigma_{A}\right|\right)^{k-1}}{d}$ fraction of the edges are bad. With high probability, the total number of edges in $G_{\alpha}$ is $\Theta\left(n \alpha \log \left|\Sigma_{A}\right|\right)$, so this means that the number of bad edges is at most $O\left(\frac{n\left(\alpha \log \left|\Sigma_{A}\right|\right)^{k}}{d}\right)$. By our choice of $\alpha$, this is at most $O(n)$.

Note that Lemmas 2.1 and 2.3 hold with high probability, whereas Lemma 3.1 holds with probability of $2 / 3$. Since we can easily calculate (in polynomial time) the number of bad edges, we can clearly repeat this sampling until we get an instance in which all three lemmas hold with high probability. Hence, we will now assume that (with high probability) $G_{\alpha}$ satisfies all three lemmas.

THEOREM 3.2. If there is no (randomized) polynomial time algorithm that can distinguish between instances of Label Cover in which $|A|=|B|=n / 2$ and all vertices have degree $d$ where all edges are satisfiable and instances where at most an $s \leq 1 /(16 \log n)$ fraction of the edges are satisfiable, then there is some constant $c$ so that for $16 \log n \leq \alpha \leq \min \left\{1 / s, d^{1 / k} / \log \left|\Sigma_{A}\right|\right\}$, there is no (randomized) polynomial time algorithm that can distinguish between instances of Label Cover ${ }_{k}$ in which all edges are satisfiable and instances in which at most a c/ $\alpha$ fraction of the edges are satisfiable.

Proof. If there is a labeling that satisfies all edges of $G$, then clearly the same labeling satisfies all edges of $G_{\alpha}^{\prime}$. On the other hand, suppose that only an $s$ fraction of the edges of $G$ can be satisfied. By Lemma 3.1, the number of bad edges is at most $O(n)$, so the total number of edges in $G_{\alpha}^{\prime}$ is still $\Theta\left(n \alpha \log \left|\Sigma_{A}\right|\right)$.

Fix any labeling of $G_{\alpha}^{\prime}$, and suppose that it satisfies a $\beta$ fraction of the edges of $G_{\alpha}^{\prime}$. Then even if it would have satisfied all of the bad edges, the number of edges of $G_{\alpha}$ that it satisfies is at most $\beta \cdot \Theta\left(n \alpha \log \left|\Sigma_{A}\right|\right)+O(n)$. By Lemma 2.1, this must be at most $(4 e / \alpha) \cdot \Theta\left(n \alpha \log \left|\Sigma_{A}\right|\right)$, and thus for some constant $c$, we have that $\beta \leq c / \alpha$.

Therefore, if we could distinguish between the case when every edge of $G_{\alpha}^{\prime}$ can be satisfied and the case when at most a $c / \alpha$ fraction can be satisfied, we could distinguish between the case when every edge of $G$ can be satisfied and the case when at most an $s$ fraction can be satisfied.

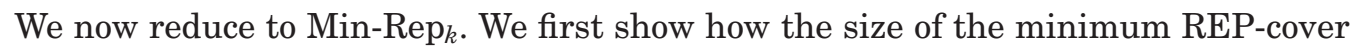
of $H_{\alpha}$ depends on $G$ (note that this proof is essentially from Kortsarz [2001] and is included here for completeness). We will then show that, similar to Label Cover, we can deterministically remove small cycles to get the instance $H_{\alpha}^{\prime}$ with large supergirth that preserves this gap.

Lemma 3.3. Let $16 \log n \leq \alpha \leq 1 /$ s. If there is a valid labeling of $G$, then the MinRep instance $H_{\alpha}$ has a REP-cover of size $n$ (where $n$ is the number of vertices in the 
supergraph). Otherwise, with high probability (at least $1-2 / n$ ), the smallest REP-cover has size at least $\Omega(n \sqrt{\alpha})$.

Proof. If there is a valid labeling of $G$, then by Lemma 2.1 there is a valid labeling of $G_{\alpha}$ (since the completeness remains 1 ), and thus there is a REP-cover of $H_{\alpha}$ of size $n$ as claimed. On the other hand, suppose that at most an $s$ fraction of the edges of $G$ can be satisfied. Then since the soundness of $G_{\alpha}$ is at most $4 e / \alpha$ by Lemma 2.1 (with high probability), any labeling of $G_{\alpha}$ satisfies at most a $4 e / \alpha$ fraction of the edges. Suppose that there is a REP-cover of $H_{\alpha}$ of size less than $n \sqrt{\alpha} /(36 \sqrt{3 e})$. We will show that this implies there is a labeling of $G_{\alpha}$ that satisfies more than a $4 e / \alpha$ fraction of the edges, giving a contradiction and proving the lemma.

Suppose that the smallest REP-cover for $H_{\alpha}$ has size $\beta n$. This means that the average number of representatives/labels assigned to each vertex in $G_{\alpha}$ by this cover is $\beta$. To analyze the labeling that covers the most edges, we analyze the random labeling obtained by choosing for each vertex a label uniformly at random from the set of labels it is assigned by the REP-cover. Let $A^{\prime} \subseteq A$ be the set of vertices in $A$ that receive at most $18 \beta$ labels in this REP-cover, and define $B^{\prime} \subseteq B$ analogously. Note that $\left|A^{\prime}\right| \geq(8 / 9)|A|$ and similarly $\left|B^{\prime}\right| \geq(8 / 9)|B|$, since otherwise the total number of representatives in the REP-cover is larger than $(1 / 9) \cdot(n / 2) \cdot(18 \beta)=\beta n$, contradicting our assumption on the size of the REP-cover. With high probability, the fraction of edges of $G_{\alpha}$ that touch a vertex of $A \backslash A^{\prime}$ is at most $\frac{(1 / 9) \cdot\left(2 \alpha \log \left|\Sigma_{A}\right|\right)}{(1 / 9) \cdot\left(2 \alpha \log \left|\Sigma_{A}\right|\right)+(8 / 9)\left(\left(\alpha \log \left|\Sigma_{A}\right|\right) / 2\right)}=1 / 3$, and similarly for the fraction of edges that touch $B \backslash B^{\prime}$ (where we used the approximate regularity from Lemma 2.3). So if we consider the subgraph of $G_{\alpha}$ induced by $A^{\prime} \cup B^{\prime}$, we still have at least $1 / 3$ of the edges of $G_{\alpha}$.

Now let $(a, b) \in A^{\prime} \times B^{\prime}$ be such an edge. Since we started with a REP-cover, there is at least one representative assigned to $a$ and one representatives assigned to $b$ that satisfy the relation $\pi_{(a, b)}$. Since both endpoints have at most $18 \beta$ representatives in the REP-cover, the probability that these two representatives are the assigned labels is at least $1 /(18 \beta)^{2}$. Thus, by linearity of expectations, we expect that at least $1 /\left(3(18 \beta)^{2}\right)=$ $1 /\left(972 \beta^{2}\right)$ fraction of the edges are covered by our random labeling, so there exists some labeling that covers at least that many. If $\beta \leq \frac{\sqrt{\alpha}}{36 \sqrt{3 e}}$, then this is at least $4 e / \alpha$, giving a contradiction. Therefore, the smallest REP-cover has size at least $(n \sqrt{\alpha}) /(36 \sqrt{3 e})$, proving the lemma.

We will now get rid of small cycles by using the instance $H_{\alpha}^{\prime}$.

THEOREM 3.4. If there is no (randomized) polynomial time algorithm that can distinguish between instances of Label Cover in which $|A|=|B|=n / 2$ and all vertices have degree $d$ where all edges are satisfiable and instances where at most an $s \leq 1 /(16 \log n)$ fraction of the edges are satisfiable, then there is some constant $c$ so that for $16 \log n \leq \alpha \leq \min \left\{1 / s, d^{1 / k} / \log \left|\Sigma_{A}\right|\right\}$, there is no (randomized) polynomial time algorithm that can distinguish between instances of Min-Rep $p_{k}$ where the smallest $R E P$-cover has size at most $n$ and instances where the smallest REP-cover has size at least $n \sqrt{\alpha} / c$ (here, $n$ is the size of the supergraph).

Proof. If there is a labeling that satisfies all edges of $G$, then clearly choosing that labeling gives a valid REP-cover of $H_{\alpha}^{\prime}$ of size at most $n$. For the other case, suppose that any labeling of $G$ satisfies at most an $s$ fraction of the edges. Then by Lemma 3.3, with high probability, the smallest REP-cover of $H_{\alpha}$ has size at least $\Omega(n \sqrt{\alpha})$. By Lemma 3.1, the number of bad edges is at most $O(n)$. Removing any particular edge (in particular a bad edge) can only decrease the size of the optimal REP-cover by at most 2, so if we remove all bad edges (getting $H_{\alpha}^{\prime}$ ), we are left with an instance with supergirth larger 
than $k$ in which the smallest REP-cover has size at least $\Omega(n \sqrt{\alpha})-O(n)=\Omega(n \sqrt{\alpha})$. By construction, the supergirth is greater than $k$, so this proves the theorem.

Now we define and analyze the PCP / Label Cover instances to which we will apply Theorems 3.2 and 3.4. Recall that Max-3SAT(5) is the problem of finding an assignment to variables of a 3-CNF formula that maximizes the number of satisfied clauses, with the additional property that every variable appears in exactly five clauses of the formula. We begin with the standard Label Cover instance for Max-3SAT(5) (e.g., see Arora and Lund [1997]). The graph $(A, B, E)$ has $|B|=n^{\prime}$ and $|A|=5 n^{\prime} / 3$ (where $n^{\prime}$ is the number of variables in the instance), and every vertex in $A$ has degree 3 and every vertex in $B$ has degree 5 . Vertices in $A$ correspond to clauses, and vertices in $B$ correspond to variables. The alphabet sizes are $\left|\Sigma_{A}\right|=7$ and $\left|\Sigma_{B}\right|=2$. The PCP theorem [Arora et al. 1998; Arora and Safra 1998] implies that the gap for these instances is constant-that is, it is hard to distinguish the case when all edges are satisfiable from the case in which $1 /(1+\epsilon)$ fraction of the edges are satisfiable, for some constant $\epsilon$.

Now we take three copies of $A$, call them $A_{1}, A_{2}, A_{3}$, and let $A^{\prime}$ be their union (so $\left.\left|A^{\prime}\right|=5 n^{\prime}\right)$. Similarly, we take five copies of $B$ to get $B_{i}$ for $i \in[5]$ and take their union to be $B^{\prime}$. Now, between each $A_{i}$ and each $B_{j}$, we put a copy of the original edge set $E$ (which we will call $E_{i j}$ ), giving us edge set $E^{\prime}$. Note that $\left|B^{\prime}\right|=\left|A^{\prime}\right|=5 n^{\prime}$ and every vertex has degree 15. Obviously, if the original instance has all edges satisfiable, then that is still true of this instance. On the other hand, suppose in the original instance that at most $1 /(1+\epsilon)$ of the edges are satisfiable. Then, fix any labeling of $A^{\prime}$ and $B^{\prime}$. For any $i, j$, this induces some labeling of $A_{i}$ and $B_{j}$, which we know can only satisfy $1 /(1+\epsilon)$ of the edges in $E_{i j}$. Since this is true for all $i, j$, the total fraction of satisfied edges is at most $1 /(1+\epsilon)$.

We now apply parallel repetition $\ell$ times. Now each side has size $\left(5 n^{\prime}\right)^{\ell}$, the degree is $d=15^{\ell}$, and the alphabet sizes are $\left|\Sigma_{A}\right|=7^{\ell}$ and $\left|\Sigma_{B}\right|=2^{\ell}$. By the parallel repetition theorem [Raz 1998], unless $N P \subseteq B P T I M E\left(n^{O(\ell)}\right)$, it is hard to distinguish between the case when all edges are satisfiable and when at most a $2^{-\ell / c}$ fraction are satisfiable for some constant $c$. We can apply Theorem 3.2 to this construction to get the following hardness result.

THEOREM 3.5. Assuming NP $\nsubseteq$ BPTIME $\left(2^{\text {polylog(n)}}\right)$, for any constant $\epsilon>0$ and $3 \leq k \leq \log ^{1-2 \epsilon} n$ there is no polynomial time algorithm that can approximate Label Cover $_{k}$ to a factor better than $2^{\left(\log ^{1-\epsilon} n\right) / k}$.

Proof. Set $\ell=\log ^{1 / \epsilon} n^{\prime}$, so the size of the Label Cover instance is $n=\left(5 n^{\prime}\right)^{\log ^{1 / \epsilon} n^{\prime}}$ and $\ell^{\epsilon}=\log n^{\prime}$. Note that $\log n=\Theta\left(\ell \log n^{\prime}\right)=\Theta\left(\ell^{1+\epsilon}\right)$, so $\ell=\Theta\left((\log n)^{1 /(1+\epsilon)}\right)$. Let $\alpha=\min \left\{2^{\ell / c}, 15^{\ell / k} / \ell \log 7\right\}$. Assuming that $k$ is at most $\log ^{(1 /(1+\epsilon))-\gamma} n$ for some constant $\gamma>0$ implies that $\alpha \geq 16 \log n$, so applying Theorem 3.2 to this construction implies that, assuming $N P \nsubseteq B P T I M E\left(n^{O(\ell)}\right)$, there is no polynomial time algorithm that can distinguish between instances of Label Cover $_{k}$ in which all edges are satisfiable and instances in which at most a $c / \alpha$ fraction are satisfiable (for some constant $c$ ). Using a smaller $\epsilon$ to change $1 /(1+\epsilon)$ to $1-\epsilon$, as well as to get rid of lower order terms, gives the theorem.

On the other hand, if we apply Theorem 3.4 to this construction then we get the following theorem.

TheOREM 3.6. Assuming NP $\nsubseteq B P T I M E\left(2^{\text {polylog(n)}}\right)$, for any constant $\epsilon>0$ and $3 \leq k \leq \log ^{1-2 \epsilon} n$ there is no polynomial time algorithm that can distinguish between

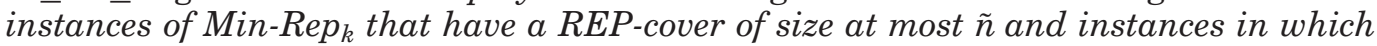


the smallest REP-cover has size at least $2^{\left(\log ^{1-\epsilon} n\right) / k} \cdot \tilde{n}$, where $n$ is the size of the Min-Rep graph and $\tilde{n}$ is the size of the supergraph. Thus, there is no polynomial time algorithm that can approximate Min-Rep $k$ to a factor better than $2^{\left(\log ^{1-\epsilon} n\right) / k}$.

Proof. As before, we set $\ell=\log ^{1 / \epsilon} n^{\prime}\left(\right.$ so $\left.\ell^{\epsilon}=\log n^{\prime}\right)$. Then $n=\left(5 n^{\prime}\right)^{\ell} \cdot 7^{\ell}+\left(5 n^{\prime}\right)^{\ell} \cdot 2^{\ell} \leq$ $2\left(35 n^{\prime}\right)^{\ell}$ is the number of vertices in the resulting Min-Rep instance. Note that, as in Label Cover, $\log n=\Theta\left(\ell \log n^{\prime}\right)=\Theta\left(\ell^{1+\epsilon}\right)$, so $\ell=\Theta\left((\log n)^{1 /(1+\epsilon)}\right)$. Applying Theorem 3.4 to this construction implies that unless $N P \subseteq B P T I M E\left(2^{\text {polylog(n) }}\right)$, there is no polynomial time algorithm that can distinguish between instances of Min-Rep with supergirth larger than $k$ where the smallest REP-cover has size at most $2\left(5 n^{\prime}\right)^{\ell}$ and instances where the smallest REP-cover has size at least $\Omega\left(\left(5 n^{\prime}\right)^{\ell} \sqrt{\min \left\{2^{\ell / c}, 15^{\ell / k} / \ell \log 7\right\}}\right.$ ) (assuming $k \leq \log ^{(1 /(1+\epsilon))-\gamma} n$ for some constant $\left.\gamma>0\right)$. Plugging in our choice of $\ell$, and using smaller values of $\epsilon$ to get rid of lower order terms and replace $1 /(1+\epsilon)$ by $1-\epsilon$, gives the theorem.

\section{HARDNESS OF BASIC $K$-SPANNER}

We now show how to reduce Min-Rep with large supergirth to the basic $k$-spanner problem. This reduction is from Elkin and Peleg [2000], which is in turn very similar to reductions from Min-Rep to other spanner problems developed by Elkin and Peleg [2007]. We include it here just for completeness and try to use their notation when possible. Suppose that we are given a Min-Rep instance with supergraph $\tilde{G}=(A, B, \tilde{E})$ with supergirth at least $k+2$, as well as the Min-Rep graph $G=\left(A \times \Sigma_{A}, B \times \Sigma_{B}, E\right)$. As before, for $i \in A$, let $A_{i}=\left\{(i, \alpha): \alpha \in \Sigma_{A}\right\}$ be the set of vertices in the Min-Rep graph corresponding to the vertex $i$ in the supergraph, and similarly for $i \in B$, let $B_{i}=\left\{(i, \beta): \beta \in \Sigma_{B}\right\}$. Let $n=|A| \cdot\left|\Sigma_{A}\right|+|B| \cdot\left|\Sigma_{B}\right|$ denote the size of the Min-Rep graph, and let $\tilde{n}=|A|+|B|$ denote the size of the supergraph. Since this instance comes from our previous reduction, we can also assume that $|A|=|B|=\tilde{n} / 2$. Let $k_{A}=\left\lfloor\frac{k-1}{2}\right\rfloor$, let $k_{B}=\left\lceil\frac{k-1}{2}\right\rceil$, and let $x=n^{2} / \tilde{n}$. To define the $k$-spanner graph $G^{\prime}$, we first define two new vertex sets:

$$
S=\left\{s_{i j}^{p}: i \in A, j \in\left[k_{A}\right], p \in[x]\right\} \text { and } T=\left\{t_{i j}^{p}: i \in B, j \in\left[k_{B}\right], p \in[x]\right\} .
$$

The vertex set of our graph $G^{\prime}$ will be $V^{\prime}=\left(A \times \Sigma_{A}\right) \cup\left(B \times \Sigma_{B}\right) \cup S \cup T$. Now we define a collection of edge sets:

$$
\begin{aligned}
E_{M}= & \left\{\left(s_{i j}^{p}, s_{i(j+1)}^{p}\right): p \in[x], i \in A, j \in\left[k_{A}-1\right]\right\}, \\
& \cup\left\{\left(t_{i j}^{p}, t_{i(j+1)}^{p}\right): p \in[x], i \in B, j \in\left[k_{B}-1\right]\right\}, \\
E_{s A}= & \left\{\left(s_{i 1}^{p}, u\right): i \in A, u \in A_{i}, p \in[x]\right\}, \\
E_{t B}= & \left\{\left(w, t_{j 1}^{p}\right): j \in B, w \in B_{j}, p \in[x]\right\}, \\
E_{\tilde{G}}^{p}= & \left\{\left(s_{i k_{A}}^{p}, t_{j k_{B}}^{p}\right): i \in A, j \in B,(i, j) \in \tilde{E}\right\}, \\
E_{\tilde{G}}= & \cup_{p=1}^{x} E_{\tilde{G}}^{p} .
\end{aligned}
$$

We let the edge set $E^{\prime}$ of $G^{\prime}$ be $E \cup E_{M} \cup E_{s A} \cup E_{t B} \cup E_{\tilde{G}}$. Note that when $k=3$, $k_{A}=k_{B}=1$, so $E_{M}$ is empty. In addition, note that for each $p \in[x]$, the edges $E_{\tilde{G}}^{p}$ form a graph isomorphic to the supergraph $\tilde{G}$.

For an edge $\left(s_{i k_{A}}^{p}, t_{j k_{B}}^{p}\right) \in E_{\tilde{G}}^{p}$, we say that a spanning path $P$ (i.e., a path from $s_{i k_{A}}^{p}$ to $t_{j k_{B}}^{p}$ of length at most $k$ ) is a canonical path if it has the form $s_{i k_{A}}^{p}, s_{i\left(k_{A}-1\right)}^{p}, \ldots$, $s_{i 1}^{p}, u_{i}, w_{j}, t_{j 1}^{p}, t_{j 2}^{p}, \ldots, t_{j k_{B}}^{p}$. In other words, the path first follows the path of $E_{M}$ edges 
to $s_{i 1}^{p}$, then uses an edge from $E_{s A}$ to get to one of the original Min-Rep nodes $u_{i}$ that corresponds to supernode $i$, then takes an original Min-Rep edge to $w_{j}$, then an $E_{t B}$ edge out to $t_{j 1}^{p}$, and then follows $E_{M}$ edges to $t_{j k_{B}}^{p}$. Note that such a path must exist or else there are no edges from $A_{i}$ to $B_{j}$, in which case $(i, j)$ would not be an edge in the supergraph, which would mean that $\left(s_{i k_{A}}^{p}, t_{j k_{B}}^{p}\right)$ would not be an edge in $E_{\tilde{G}}^{p}$. Furthermore, any canonical path has length exactly $\left(k_{A}-1\right)+1+1+1+\left(k_{B}-1\right)=k$, so it is indeed a valid spanning path.

Lemma 4.1. In any $k$-spanner $H$ of $G^{\prime}$, every edge in $E_{\tilde{G}}$ is either included in $H$ or is spanned by a canonical path.

Proof. Suppose that this is false, and let $e=\left(s_{i k_{A}}^{p}, t_{j k_{B}}^{p}\right)$ be an edge in $E_{\tilde{G}}$ that is not in $H$ but is also not spanned by a canonical path. Let $P=\left(s_{i k_{A}}^{p}=y_{1}, y_{2}, \ldots, y_{q-1}, y_{q}=t_{j k_{B}}^{p}\right)$ be the shortest simple path in $H$ that does span $e$ (such a path with $q \leq k+1$ must exist since $H$ is a $k$-spanner of $\left.G^{\prime}\right)$. Let $U=\left\{s_{i^{\prime} k_{A}}^{p}: i^{\prime} \in A\right\} \cup\left\{t_{j^{\prime} k_{B}}^{p}: j^{\prime} \in B\right\}$ be the set of vertices that are incident on edges of $E_{\tilde{G}}^{p}$. Let $y_{\alpha}$ be the first vertex in $P$ that is not in $U$. Such a vertex must exist, because if it does not, then $P$ is a path of length at most $k$ between $s_{i k_{A}}^{p}$ and $t_{j k_{B}}^{p}$ that is contained in $E_{\tilde{G}}^{p}$. This is a contradiction, as $E_{\tilde{G}}^{p}$ is isomorphic to the supergraph $\tilde{G}$, which by assumption has girth at least $k+2$, whereas adding $e$ to $P$ would give a cycle of length at most $k+1$.

Thus, $y_{\alpha}$ is the first vertex in $P$ that is not in $U$. This means that it must be either $s_{i^{\prime}\left(k_{A}-1\right)}^{p}$ or $t_{j^{\prime}\left(k_{B}-1\right)}^{p}$ for some $i^{\prime} \in A$ or $j^{\prime} \in B$ (unless $k=3$ or 4 , in which case it must be a node in $A_{i^{\prime}}$ or $\left.B_{j^{\prime}}\right)$. If it is $t_{j^{\prime}\left(k_{B}-1\right)}^{p}$, then $P$ must keep following $E_{M}$ edges to get that $y_{\alpha+k_{B}-2}$ is $t_{j^{\prime} 1}^{p}$, and therefore $y_{\alpha+k_{B}-1}$ is $w$ for some $w \in B_{j^{\prime}}$. Since $\alpha \geq 2$ and $k_{A}+k_{B}=k-1$, there can be only $k_{A}+1$ more vertices on the path. But it is obvious that if $j^{\prime} \neq j$ (which it must be, since otherwise the path would have already hit $t_{j k_{B}}^{p}$ ), there is no way to complete the path.

If $y_{\alpha}=s_{i^{\prime}\left(k_{A}-1\right)}^{p}$, then since the intermediate vertices have degree 2 and $P$ is simple, it must be the case that $y_{\alpha+k_{A}-2}$ is $s_{i^{\prime} 1}^{p}$, and so $y_{\alpha+k_{A}-1}$ is $u$ for some $u \in A_{i^{\prime}}$. From $u$, the next vertex could either be $s_{i^{\prime} 1}^{p^{\prime}}$ for some other $p^{\prime}$ or $w \in B_{j^{\prime}}$ for some $j^{\prime} \in B$. If $y_{\alpha+k_{A}}$ is $s_{i^{\prime} 1}^{p^{\prime}}$ for some $p^{\prime}$, then the next hop cannot be to a node in $A_{i^{\prime}}$, or else we could have gotten to this node instead of to $u$ earlier, contradicting our assumption that $P$ is the shortest path. Thus, if $y_{\alpha+k_{A}}$ is $s_{i^{\prime} 1}^{p^{\prime}}$ for some $p^{\prime}$, it must be that $P$ follows $E_{M}$ edges backward to get that $y_{\alpha+2 k_{A}-1}=s_{i^{\prime} k_{A}}^{p^{\prime}}$. Note that $\alpha \geq 2$ by definition, and $2 k_{A} \geq k-2$, so either $y_{k}$ or $y_{k-1}$ is $s_{i^{\prime} k_{A}}^{p^{\prime}}$. Either one is an obvious contradiction, as $y_{k+1}$ is supposed to be $t_{j k_{B}}^{p}$, which is not within distance two from $s_{i^{\prime} k_{A}}^{p^{\prime}}$.

This means that from $u$, the next vertex in $P$ must be $w \in B_{j^{\prime}}$ for some $j^{\prime} \in B$, or equivalently that $y_{\alpha+k_{A}}=w$. Since $\alpha \geq 2$ and $k_{A}+k_{B}=k-1$, there can be at most $k_{B}$ more vertices on $P$. To get to $t_{j k_{B}}^{p}$ via $E_{M}$ edges, it obviously must be the case that $j^{\prime}=j$ and $P$ is actually a canonical path. Otherwise, if the next hop from $w$ is another edge from $E$, then it is back on the $A$ side of the Min-Rep graph, which is clearly too far away from $t_{j k_{B}}^{p}$ to finish the path. Thus, $P$ must actually be a canonical path.

We will now define some edge sets that will be useful in the next lemma. For each $i \in A$, let $u_{i} \in A_{i}$ be some arbitrarily chosen vertex in $A_{i}$, and let $\hat{E}_{i}=\left(\cup_{u \in A_{i}}\left(s_{i 1}^{1}, u\right)\right) \cup$ $\left(\cup_{p \in[x]}\left(s_{i 1}^{p}, u_{i}\right)\right)$. Similarly, for each $j \in B$, we arbitrarily choose some node $w_{j} \in B_{j}$, 
and let $\hat{E}_{j}=\left(\cup_{w \in B_{j}}\left(w, t_{j 1}^{1}\right)\right) \cup\left(\cup_{p \in[x]}\left(w_{j}, t_{j 1}^{p}\right)\right)$. Let $\hat{E}=\left(\cup_{i \in A} \hat{E}_{i}\right) \cup\left(\cup_{j \in B} \hat{E}_{j}\right)$. Clearly, $|\hat{E}| \leq n+x \tilde{n}$, since $\left|\hat{E}_{i}\right|=\left|A_{i}\right|+x-1$ for each $i \in A$ and $\left|\hat{E}_{j}\right|=\left|B_{j}\right|+x-1$ for each $j \in B$.

We say that a spanner $H$ of $G^{\prime}$ is a proper $k$-spanner if it does not include any edge of $E_{\tilde{G}}$, which by Lemma 4.1 implies that every edge of $E_{\tilde{G}}$ is spanned by a canonical path.

Lemma 4.2. Any $k$-spanner $H$ for $G^{\prime}$ can be converted in polynomial time into a proper $k$-spanner $H^{\prime}$ of size at most $6|H|$.

Proof. We first let $H_{1}$ be the edge set $\left(H \backslash E_{\tilde{G}}\right) \cup E \cup E_{M} \cup \hat{E}$. It is obvious that all edges of $G^{\prime}$ are $k$-spanned by $H_{1}$ except for $H \cap E_{\tilde{G}}$ : edges in $E, E_{M}$, and $\hat{E}$ are self-spanned, edges in $E_{s A}$ and $E_{t B}$ are 3-spanned by $\hat{E}$, and edges in $E_{\tilde{G}} \backslash H$ must have been spanned in $H$ by a canonical path (by Lemma 4.1 ), which is still contained in $H_{1}$. We now claim that $H_{1}$ is small. Note that $\left|V^{\prime}\right|=n+x(\tilde{n} / 2) k_{A}+x(\tilde{n} / 2) k_{B}=n+x(\tilde{n} / 2)(k-1)$. So

$$
\begin{aligned}
\left|H_{1}\right| & \leq|H|+|E|+\left|E_{M}\right|+|\hat{E}| \\
& \leq|H|+n^{2}+x(\tilde{n} / 2)\left(k_{A}-1\right)+x(\tilde{n} / 2)\left(k_{B}-1\right)+n+\tilde{n} x \\
& \leq|H|+x \tilde{n}+x(\tilde{n} / 2)(k-3)+n+\tilde{n} x \\
& \leq|H|+\left(\left|V^{\prime}\right|-1\right)+\left(\left|V^{\prime}\right|-1\right)+\left(\left|V^{\prime}\right|-1\right) \\
& \leq 4|H|,
\end{aligned}
$$

where we used the fact that $k \geq 3$ and the fact that $|H| \geq\left|V^{\prime}\right|-1$ (since it is connected).

Now we need to span the edges in $H \cap E_{\tilde{G}}$. For each such edge $\left(s_{i k_{A}}^{p}, t_{j k_{B}}^{p}\right)$, there is an associated superedge $(i, j)$. We get $H^{\prime}$ from $H_{1}$ by adding, for each such edge, the edges $\left(s_{i 1}^{p}, u\right)$ and $\left(w, t_{j 1}^{p}\right)$ for some $u \in A_{i}$ and $w \in B_{j}$ so that $(u, w) \in E$ (note that some such edge must exist or else $\pi_{(i, j)}$ is empty). This obviously creates a canonical path that spans $\left(s_{i k_{A}}^{p}, t_{j k_{B}}^{p}\right)$ (since $E_{M} \subseteq H_{1}$ ) while costing at most $2|H|$, and thus $H^{\prime}$ is a valid $k$-spanner of size at most $6|H|$ as claimed.

We now can prove one direction of the reduction.

LEMMA 4.3. Given a $k$-spanner $H$ for $G^{\prime}$, we can construct in polynomial time a REP-cover for $G$ of size at most $6|H| / x$.

Proof. We first apply Lemma 4.2 to get a proper $k$-spanner $H^{\prime}$ of size at most $6|H|$. Now, for every $p \in[x]$ and $i \in A$, let $S_{i}^{p}=\left\{u \in A_{i}:\left(s_{i 1}^{p}, u\right) \in E\left(H^{\prime}\right)\right\}$, and similarly for $j \in B$, let $T_{j}^{p}=\left\{w \in B_{j}:\left(w, t_{j 1}^{p}\right) \in E\left(H^{\prime}\right)\right\}$. Now, for each $p \in[x]$, let $U^{p}=\left(\cup_{i \in A} S_{i}^{p}\right) \cup\left(\cup_{j \in B} T_{j}^{p}\right)$. We claim that for every $p \in[x]$, the set $U^{p}$ is a valid REPcover. This is enough to prove the lemma, since clearly the smallest $U^{p}$ has size at most $6|H| / x$ by averaging (each vertex in each $U^{p}$ can be charged to the edge in $E_{s A}$ or $E_{t B}$ that caused it to be in $\left.U^{p}\right)$.

Consider a superedge $(i, j) \in \tilde{E}$. Since $H^{\prime}$ is a proper $k$-spanner, it contains a canonical path from $s_{i k_{A}}^{p}$ to $t_{j k_{B}}^{p}$. By the definition of canonical path, this implies that it includes the edges $\left(s_{i 1}^{p}, u\right),(u, w)$, and $\left(w, t_{j 1}^{p}\right)$ for some $u \in A_{i}$ and $w \in B_{j}$. Thus, $u \in S_{i}^{p}$ and $w \in T_{j}^{p}$ with $(u, w) \in E$. Since this is true for every superedge, it implies that $U^{p}$ is a valid REP-cover.

We now want to prove the other direction-that the existence of a small REP-cover for $G$ implies a small $k$-spanner for $G^{\prime}$.

LEMMA 4.4. Given a REP-cover $C$ for $G$, we can construct in polynomial time a $k$-spanner $H$ of $G^{\prime}$ with at most $(k+1) x|C|$ edges. 
Proof. The edge set of our $k$-spanner $H$ is

$\left\{\left(s_{i 1}^{p}, u\right): i \in A, u \in A_{i} \cap C, p \in[x]\right\} \cup\left\{\left(t_{j 1}^{p}, w\right): j \in B, w \in B_{j} \cap C, p \in[x]\right\} \cup E \cup E_{M} \cup \hat{E}$.

To see that $H$ is a $k$-spanner, first note that every edge in $E \cup E_{M} \cup \hat{E}$ is spanned by itself. It is easy to see that any edge in $E_{s A}$ or $E_{t B}$ is spanned by $\hat{E}$ (in fact, 3 -spanned). And since $C$ is a valid REP-cover, for any edge in $E_{\tilde{G}}$ there is a canonical path included in $H$.

Now we need to bound the size of $H$. Clearly, it is at most

$$
\begin{aligned}
|H| & =x|C|+|E|+\left|E_{M}\right|+|\hat{E}| \\
& \leq x|C|+n^{2}+x|A|\left(k_{A}-1\right)+x|B|\left(k_{B}-1\right)+(n+x \tilde{n}) \\
& \leq x|C|+x \tilde{n}+x(\tilde{n} / 2)\left(k_{A}-1\right)+x(\tilde{n} / 2)\left(k_{B}-1\right)+n+x \tilde{n} \\
& \leq x|C|+x|C|+x(\tilde{n} / 2)(k-3)+x \tilde{n}+x \tilde{n} \\
& \leq 4 x|C|+x|C| \frac{k-3}{2} \\
& \leq(k+1) x|C|,
\end{aligned}
$$

where we used the fact that $|C| \geq \tilde{n}$ and that by definition $x=n^{2} / \tilde{n}$, as well as the fact that $k \geq 3$.

We can now prove the main theorem of the article- that it is hard to approximate the basic $k$-spanner problem.

THEOREM 4.5. Assuming NP $\nsubseteq$ BPTIME $\left(2^{\text {polylog(n)}}\right)$, for any constant $\epsilon>0$ and $3 \leq k \leq \log ^{1-2 \epsilon} n$ there is no polynomial time approximation algorithm for the basic $k$-spanner problem with ratio less than $2^{\left(\log ^{1-\epsilon} n\right) / k}$.

Proof. Suppose that we have an $\alpha$-approximation algorithm for the basic $k$-spanner problem. Given an instance $G$ of Min-Rep with supergirth larger than $k+1$, we reduce it to basic $k$-spanner on $G^{\prime}$ as described earlier. If the smallest REP-cover has size $\tilde{n}$ (i.e., we can assign a single label to every vertex of the supergraph and get a valid proof), then by Lemma 4.4 there is a $k$-spanner of $G^{\prime}$ with at most $(k+1) x \tilde{n}$ edges. On the other hand, if the smallest REP-cover has size at least $2^{\left(\log ^{1-\epsilon} n\right) /(k+1)} \cdot \tilde{n}$, then by Lemma 4.3 the smallest $k$-spanner of $G^{\prime}$ must have size at least $2^{\left(\log ^{1-\epsilon} n\right) /(k+1)} \cdot \tilde{n} x / 6$. By Theorem 3.6, we cannot distinguish between these two cases of Min-Rep, and thus $\alpha \geq\left(2^{\left(\log ^{1-\epsilon} n\right) /(k+1)} \tilde{n} x / 6\right) /((k+1) x \tilde{n})=\left(2^{\left(\log ^{1-\epsilon} n\right) /(k+1)}\right) /(6(k+1))$.

However, the $n$ used in the preceding expression is the size of the Min-Rep instance $G$, not the size of the spanner instance $G^{\prime}$. Let $n^{\prime}=\left|V^{\prime}\right|$ be the size of the $k$-spanner instance, and note that $n^{\prime} \leq n+\tilde{n} k x=n+n^{2} k \leq 2 k n^{2}$. So $n \geq \sqrt{n^{\prime} /(2 k)}$, and thus we have hardness

$$
\frac{2^{\left(\log ^{1-\epsilon} n\right) /(k+1)}}{6(k+1)} \geq \frac{2^{\left(\log ^{1-\epsilon}\left(n^{\prime} /(2 k)\right)\right) /(2(k+1))}}{6(k+1)} .
$$

By using an appropriately smaller $\epsilon$, and switching notation to let $n$ represent the size of the $k$-spanner instance, this gives hardness of $2^{\left(\log ^{1-\epsilon} n\right) / k}$ as claimed (assuming that $\left.k \leq \log ^{1-2 \epsilon} n\right)$. 


\section{DISCUSSION AND OPEN PROBLEMS}

Motivated by proving hardness for the basic $k$-spanner problem (one of the only spanner problems for which strong hardness was not already known), we gave a proof that Label Cover and Min-Rep are hard to approximate even when restricted to instances with large supergirth. This result has been claimed before in Elkin and Peleg [2000], but their proof was fundamentally flawed by their attempt to increase the girth before using parallel repetition. Our new proof is based on a technique (subsampling edges of 2-query PCPs) that allows us to sparsify the PCP obtained by parallel repetition enough to destroy most small cycles without significantly losing in the soundness of the PCP (and thus the provable hardness). This gives a proof that the basic $k$-spanner problem, which is perhaps the simplest of spanner problems, has super-polylogarithmic hardness.

An important but perhaps difficult question is if Min-Rep is still hard to approximate on instances with large girth (even if the supergirth is 4). A solution to this question would be useful in lower bounding problems such as multicommodity buy-at-bulk, multicommodity cost-distance (see Chekuri et al. [2010]), and other network design problems, and would also lead to simplifications of already known lower bounds.

New directions? In Berman et al. [2013], the directed spanner problem is studied and an $O(\sqrt{n} \log n)$ approximation is provided. Is it possible to provide an $\Omega(\sqrt{n})$ lower bound? In addition, new variants of the spanner problem have recently emerged, such as fault-tolerant spanners (see Dinitz and Krauthgamer [2011b]) and transitive closure spanners (see Raskhodnikova [2010]). The many open questions on approximatingthese and other variants of spanners may be a nice research direction for the future.

\section{REFERENCES}

Ingo Althöfer, Gautam Das, David Dobkin, Deborah Joseph, and José Soares. 1993. On sparse spanners of weighted graphs. Discrete and Computational Geometry 9, 1, 81-100. DOI:http://dx.doi.org/ 10.1007/BF02189308

Sanjeev Arora and Carsten Lund. 1997. Hardness of approximations. In Approximation Algorithms for NP-Hard Problems, D. Hochbaum (Ed.). PWS Publishing, Boston, MA, 399-446.

Sanjeev Arora, Carsten Lund, Rajeev Motwani, Madhu Sudan, and Mario Szegedy. 1998. Proof verification and the hardness of approximation problems. Journal of the ACM 45, 3, 501-555.

Sanjeev Arora and Shmuel Safra. 1998. Probabilistic checking of proofs: A new characterization of NP. Journal of the ACM 45, 1, 70-122.

Baruch Awerbuch, Alan Baratz, and David Peleg. 1992. Efficient Broadcast and Light Weight Spanners. Technical Report CS92-22. Weizman Institute of Science, Rehovot, Israel.

Hans-Jrgen Bandelt and Andreas Dress. 1986. Reconstructing the shape of a tree from observed dissimilarity data. Advances in Applied Mathematics 7, 3, 309-343.

Surender Baswana and Sandeep Sen. 2006. Approximate distance oracles for unweighted graphs in expected $\mathrm{O}\left(n^{2}\right)$ time. ACM Transactions on Algorithms 2, 4, 557-577.

Piotr Berman, Arnab Bhattacharyya, Konstantin Makarychev, Sofya Raskhodnikova, and Grigory Yaroslavtsev. 2013. Approximation algorithms for spanner problems and directed Steiner forest. Information and Computation 222, 93-107. DOI : http://dx.doi.org/10.1016/j.ic.2012.10.007

Arnab Bhattacharyya, Elena Grigorescu, Kyomin Jung, Sofya Raskhodnikova, and David P. Woodruff. 2009 Transitive-closure spanners. In Proceedings of the 20th Annual ACM-SIAM Symposium on Discrete Algorithms (SODA'09). 932-941.

Chandra Chekuri, Mohammad Taghi Hajiaghayi, Guy Kortsarz, and Mohammad R. Salavatipour. 2010. Approximation algorithms for nonuniform buy-at-bulk network design. SIAM Journal on Computing $39,5,1772-1798$.

Edith Cohen. 1999. Fast algorithms for constructing $t$-spanners and paths with stretch $t$. SIAM Journal on Computing 28, 1, 210-236.

Edith Cohen. 2000. Polylog-time and near-linear work approximation scheme for undirected shortest paths. Journal of the ACM 47, 1, 132-166.

Lenore Cowen. 2001. Compact routing with minimum stretch. Journal of Algorithms 38, 1, 170-183. 
Lenore Cowen and Christopher G. Wagner. 2004. Compact roundtrip routing in directed networks. Journal of Algorithms 50, 1, 79-95.

Michael Dinitz and Robert Krauthgamer. 2011a. Directed spanners via flow-based linear programs. In Proceedings of the 43rd Annual ACM Symposium on Theory of Computing (STOC'11). ACM, New York, NY, 323-332.

Michael Dinitz and Robert Krauthgamer. 2011b. Fault-tolerant spanners: Better and simpler. In Proceedings of the 30th Annual ACM SIGACT-SIGOPS Symposium on Principles of Distributed Computing (PODC'11). ACM, New York, NY, 169-178.

Devdatt Dubhashi and Alessandro Panconesi. 2009. Concentration of Measure for the Analysis of Randomized Algorithms. Cambridge University Press, New York, NY.

Michael Elkin. 2005. Computing almost shortest paths. ACM Transactions on Algorithms 1, 2, $283-323$.

Michael Elkin and David Peleg. 2000. Strong inapproximability of the basic $k$-spanner problem. In Proceedings of the 27th International Colloquium on Automata, Languages, and Programming (ICALP'00). 636-647.

Michael Elkin and David Peleg. 2005. Approximating $k$-spanner problems for $k>2$. Theoretical Computer Science 337, 1-3, 249-277. DOI : http://dx.doi.org/10.1016/j.tcs.2004.11.022

Michael Elkin and David Peleg. 2007. The hardness of approximating spanner problems. Theory of Computing Systems 41, 4, 691-729.

Uriel Feige. 1998. A threshold of $\ln n$ for approximating set cover. Journal of the ACM 45, 4, 634-652.

Uriel Feige and Joe Kilian. 1998. Zero knowledge and the chromatic number. Journal of Computer and System Sciences 57, 2, 187-199.

Joan Feigenbaum, Sampath Kannan, Andrew McGregor, Siddharth Suri, and Jian Zhang. 2008. Graph distances in the data-stream model. SIAM Journal on Computing 38, 5, 1709-1727.

Oded Goldreich and Madhu Sudan. 2006. Locally testable codes and PCPs of almost-linear length. Journal of the ACM 53, 4, 558-655. DOI : http://dx.doi.org/10.1145/1162349.1162351

Johan Håstad. 2001. Some optimal inapproximability results. Journal of the ACM 48, 4, 798-859.

Subhash Khot. 2005. On the unique games conjecture. In Proceedings of the 46th Annual IEEE Symposium on Foundations of Computer Science (FOCS'05). Los Alamitos, CA, 3.

Guy Kortsarz. 2001. On the hardness of approximating spanners. Algorithmica 30, 3, 432-450.

Guy Kortsarz and David Peleg. 1994. Generating sparse 2-spanners. Journal of Algorithms 17, 2, $222-236$.

Bundit Laekhanukit. 2014. Parameters of two-prover-one-round game and the hardness of connectivity problems. In Proceedings of the 25th Annual ACM-SIAM Symposium on Discrete Algorithms (SODA'14). 1626-1643. http://dl.acm.org/citation.cfm?id=2634074.2634192.

Christos Levcopoulos and Andrzej Lingas. 1992. There are planar graphs almost as good as the complete graphs and almost as cheap as minimum spanning trees. Algorithmica 8, 3, 251-256.

Carsten Lund and Mihalis Yannakakis. 1994. On the hardness of approximating minimization problems. Journal of the ACM 41, 5, 960-981.

David Peleg and Alejandro Schaffer. 1989. Graph spanners. Journal of Graph Theory 13, 99-116.

David Peleg and Jeffrey D. Ullman. 1989. An optimal synchronizer for the hypercube. SIAM Journal on Computing 18, 4, 740-747.

David Peleg and Eli Upfal. 1989. A trade-off between space and efficiency for routing tables. Journal of the ACM 36, 3, 510-530. DOI : http://dx.doi.org/10.1145/65950.65953

Sofya Raskhodnikova. 2010. Transitive-closure spanners: A survey. In Property Testing, O. Goldreich (Ed.). Springer-Verlag, Berlin, Germany, 167-196. http://dl.acm.org/citation.cfm?id=1986603.1986615.

Ran Raz. 1998. A parallel repetition theorem. SIAM Journal on Computing 27, 3, 763-803.

Iam Roditty, Mikkel Thorup, and Uri Zwick. 2008. Roundtrip spanners and roundtrip routing in directed graphs. ACM Transactions on Algorithms 4, 3, Article No. 29.

Mikkel Thorup and Uri Zwick. 2001. Compact routing schemes. In Proceedings of the 13th Annual ACM Symposium on Parallel Algorithms and Architectures (SPAA'01). ACM, New York, NY, 1-10.

Mikkel Thorup and Uri Zwick. 2005. Approximate distance oracles. Journal of the ACM 52, 1, 1-24.

Received March 2013; accepted August 2015 